Cinémas

Revue d'études cinématographiques

Journal of Film Studies

\title{
METZ, Christian. L'Énonciation impersonnelle ou le site du film. Paris : Méridiens Klincksieck, 1991, 228 p.
}

\section{Jean Châteauvert}

Volume 3, numéro 2-3, printemps 1993

URI : https://id.erudit.org/iderudit/1001201ar

DOI : https://doi.org/10.7202/1001201ar

Aller au sommaire du numéro

Éditeur(s)

Cinémas

ISSN

1181-6945 (imprimé)

1705-6500 (numérique)

Découvrir la revue

Citer ce compte rendu

Châteauvert, J. (1993). Compte rendu de [METZ, Christian. L’Énonciation impersonnelle ou le site du film. Paris : Méridiens Klincksieck, 1991, 228 p.]

Cinémas, 3(2-3), 241-246. https://doi.org/10.7202/1001201ar d'utilisation que vous pouvez consulter en ligne.

https://apropos.erudit.org/fr/usagers/politique-dutilisation/ 
METZ, Christian. L'Énonciation impersonnelle ou le site du film. Paris : Méridiens Klincksieck, 1991, 228p.

En 1987, Christian Metz publiait, dans la revue Vertigo $^{1}$, un article dans lequel il avançait une conception de l'énonciation filmique qui rompait avec le modèle littéraire : il y dénonçait la conception anthropomorphe de l'énonciation au cinéma et proposait une structure avec deux actants, le foyer et la cible de l'énonciation. L'Énonciation impersonnelle ou le site du film est le résultat de trois années d'approfondissement mené par Metz dans le cadre de son séminaire de recherche. Il y développe la réflexion entreprise sur l'énonciation filmique par l'analyse de ce qu'il appelle «différents paysages de l'énonciation», soit différentes formes que revêt l'énonciation dans le matériel filmique.

Les travaux sur l'énonciation au cinéma ont longtemps été un «placage» des catégories linguistiques. C'est sur cet arrière-fond théorique que Metz s'appuie pour donner le coup d'envoi à sa recherche : a-t-on intérêt à se fonder sur un modèle énonciatif déictique pour penser l'énonciation au cinéma (p. 17)? En soulevant cette question, Metz se fait l'écho des principales critiques qui ont été adressées aux modèles énonciatifs, notamment par David Bordwell2, c'est-à-dire le placage linguistique à un médium qui est dénué de je / tu et qui est, par sa matérialité, monodirectionnel. La réponse à ces critiques est l'occasion d'une première mise au point sur laquelle s'appuiera Metz pour conduire son analyse :

Qu'est-ce que l'énonciation au fond? Ce n'est pas forcément toujours "JE-ICI-MAINTENANT», c'est de façon plus générale la capacité qu'ont beaucoup d'énoncés à se plisser par endroits, à apparaître ici ou là, comme en relief, à se desquamer d'une fine pellicule d'eux-mêmes qui porte gravées quelques indications d'une autre nature (ou d'un autre niveau), concernant la production et non le produit (...). L'énonciation est l'acte sémiologique par lequel certaines parties du texte nous parlent de ce texte comme d'un acte (p. 20).

Conséquence de cette rupture avec le modèle linguistique, l'énonciateur devient le film lui-même en tant qu'il est orienté (p. 26), en tant qu'il est une activité qui sous-tend chaque fragment du film (p. 36). L'apport capital de cette thèse tient à ce que l'énonciation cesse d'être un dialogue entre un «je» et un 
«tu» et se pose comme une orientation métadiscursive qui se veut à la fois le reflet et / ou un commentaire sur le film.

À partir de cette nouvelle conception de l'énonciation filmique, Metz entreprend une recherche sous forme «d'une visite guidée de quelques paysages de l'énonciation filmique», ou plus exactement de dix figures et de leur variantes qui dévoilent, chacune à sa façon, l'énonciation.

La première figure correspond à la voix d'adresse dont le locuteur figure à l'image; tantôt jumelée à un regard à la caméra, l'adresse peut être destinée à un public diégétique, qui tient lieu de relais au regard sur l'espace spectatoriel; tantôt sous forme de conteur explicite comme dans le documentaire, l'adresse peut ponctuer ça et là le cours du film. On ne saurait confondre ce personnage visualisé avec l'énonciation filmique : le statut énonciatif du personnage visualisé est toujours déjà diégétique, inclus dans le film (p. 50). De façon corollaire, le «vous» désigné par le discours n'est jamais le spectateur quoique ce dernier soit assuré que, dans cette figure énonciative, on parle pour lui (p. 51) : c'est un «vous» aveugle et indéterminé mais fondamentalement disponible pour le spectateur.

La seconde figure correspond à ce Michel Chion nomme la voix $-\mathrm{Je}^{3}$, à la voix de commentateur et aux autres sons apparentés, soit les occurrences sonores dont l'ancrage visuel est absent de l'image au moment où ils sont entendus (p. 55). La voix-Je, entendre le narrateur homodiégétique, occuperait un statut particulier qui la placerait en quelque sorte en marge de la diégèse visualisée d'où un statut juxtadiégétique qui lui conférerait un «coefficient d'adresse» (p. 57) susceptible de varier depuis le faible écho d'adresse jusqu'au discours explicitement tourné vers le spectateur (p. 58). La voix du commentateur, c'est-à-dire le narrateur hétérodiégétique, a d'emblée un statut en marge de l'histoire racontée : elle entoure l'histoire, tantôt s'en approche, tantôt s'en éloigne mais toujours en lui demeurant extérieur, d'où son statut péridiégétique. La musique d'accompagnement et certains bruits qui échappent à la diégèse visualisée bénéfieraient eux aussi d'un certain coefficient d'adresse, d'une certaine propension à être "pour le spectateur» par rapport, par exemple, aux dialogues, bruits, sons qui participent du monde visualisé (p. 60).

Les adresses écrites et cartons d'adresse présentent eux aussi des forces énonciatives des plus diverses, qui peuvent varier depuis une valeur informative (ils situent un personnage, un lieu, etc.) jusqu'à la force interpellative forte (l'utilisation de la seconde personne, le ton impératif, etc.). Et Metz de souligner à 
ce chapitre que les génériques ont, par leur statut pragmatique, une certaine force énonciative (p. 68). L'analyse conduite en terme de force énonciative et non pas en terme d'une opposition énoncé-énonciation m'apparaît tout à fait symptomatique de l'orientation de cette recherche.

Les écrans seconds sont des formes de restrictions imposées dans le champ visuel, qui permettent au film de redoubler le cadrage lié à sa propre matérialité (p. 71). L'écran second peut prendre la forme d'un véritable surcadrage lorsqu'il met en scène un écran secondaire comme il peut prendre la forme de procédés techniques qui traduisent plus ou moins parfaitement le dispositif (effets de rideaux, de scène, de volets, etc.). Par rapport aux procédés examinés plus haut qui se présentent comme une forme de commentaire énonciatif, l'écran second correspond au deuxième grand registre de l'énonciation filmique, la forme réflexive : le film offre en quelque sorte un portrait de lui-même (p.73).

De la même façon, les jeux de miroirs sont souvent l'occasion de reproduire, au sein du film, son contexte énonciatif. Ainsi, le miroir peut présenter un cadre dans le cadre, il peut servir à multiplier les regards, modeler l'espace, créer des effets de suspense comme il peut se présenter comme l'écran d'un autre film lorsque la réflexion ouvre sur un monde autre (pp. 80-83).

Metz dénonce le caractère illusoire du procédé qui consiste à «montrer le dispositif»: dans la plupart des cas, ce qui est montrer n'est pas le dispositif du film mais un dispositif cinématographique mis en scène (p. 87). Metz remarque aussi que si certains films présentent une véritable analyse de leur dispositif, la majorité offre une monstration du dispositf cinématographique (p. 88). De là, il distingue 4 modes de présentation du dispositif : il peut apparaître comme figure emblématique du cinéma, prendre la forme d'une présence sensible qui colore subrepticement le film, adopter des formes très diverses qui offrent une parenté thématique ou encore, cas ultime, dans certains films qui dévoilent leurs propres artifices, figurer explicitement dans le corps du film (p. 89).

Le film dans le film est l'occasion d'une véritable mise en abyme pour l'énonciation filmique. Cette figure peut encore une fois varier depuis la forme d'un film isolé et isolable au sein d'un autre film (qui peut à l'occasion prendre l'aspect d'une citation, d'un clin d'œil) jusqu'à la forme d'un redoublement parfait dans lequel un film se tisse à travers un autre film (pp. 94-98). Suivant Metz, la pièce de théâtre, le vidéo, le flash-back par son caractère autonome et découpé et même le remake en tant qu'il 
est sous-tendu par un autre film seraient autant de formes de mise en abyme de l'énonciation filmique, autant d'occasion pour un film de se réflechir dans la texture filmique.

Le chapitre sur les images subjectives et sur le point de vue est l'occasion de mises au point tout à fait capitales pour la focalisation. Faisant grand état des travaux de Branigan ${ }^{4}$, Jost ${ }^{5}$ et Vernet ${ }^{6}$, Metz réexamine les distinctions entre les différents modes de focalisation, visuelle, cognitive, et auditive et propose une série de précisons qui exigeraient, à elle seules, un compte rendu substantiel, extrêmement intéressantes pour l'étude de la focalisation (p. 120).

La voix-Je qui correspond au narrateur homodiégétique en voix over peut avoir des statuts très divers : la voix peut correspondre à la pensée ou aux paroles du personnage comme ce peut être l'occasion, pour le film, de s'exprimer à travers un personnage (p. 138). Une dimension transversale cependant : la voix-Je marque toujours une relation d'attribution d'un fragment du film à un personnage (p. 138). C'est son coefficient d'adresse ou plus exactement de semi-adresse, l'impression qu'elle est destinée au spectateur qui la place à la périphérie de la diégèse, en position juxtadiégétique (p. 140).

L'étude du régime objectif orienté est l'occasion d'une importante mise au point qui permet de tracer une frontière entre le style d'un auteur et l'énonciation filmique. Le premier se définit par son caractère personnel, son omniprésence insaisissable, ses formes concrètes, tantôt stylistiques tantôt de contenu, et figure comme une teinte dans le corps du film. L'énonciation, à l'inverse, est d'emblée impersonnelle, occupe des parcours balisés, prend les traits d'une construction logique et reste toujours en marge du discours (pp. 158-159). Metz souligne au sujet du régime objectif orienté son caractère labile, sa propension à se confondre avec le style précisément mais aussi avec la subjectivité des personnages (p. 159).

En marge de tous ces paysages énonciatifs, on retrouve l'idée de l'image et du son neutres par rapport auxquels se définiraient les différentes marques énonciatives. Metz souligne que pareille catégorie n'existe pas, qu'elle est pure fabulation qui a toutefois son utilité en ce qu'elle permet de dessiner une limite théorique, une tendance possible pour l'énonciation filmique. L'énonciation n'est alors pas absente, mais demeure en arrière-fond, ne seraitce que comme présupposée au film.

Le dernier volet du travail de recherche de Metz, intitulé (cinéphilie oblige) : «Quatre pas dans les nuages», se présente comme trois mises au point déterminantes dans une réflexion sur 
l'énonciation filmique. La première concerne les énoncés «transparents» que Metz dénonce comme une fiction que l'illusion référentielle et le travail de certains films contribuent à créer (p. 178) : la transparence ne peut jamais qu'être une impression spectatorielle construite par le film.

Le second volet se veut une réponse aux critiques de David Bordwell et à celles de Gérard Genette, le premier reprochant aux travaux sur l'énonciation cinématographique de faire du placage linguistique, le second de poser des correspondances indues entre film et roman, l'un représentant un monde, l'autre le racontant (p. 181). La réponse à Genette tient dans le constat que le rapprochement de deux images décale le film vers le roman, le raccord étant déjà de l'ordre discursif (p. 182). La réponse à Bordwell est beaucoup plus élaborée. Quoique la narration et l'énonciation soient deux dimensions différentes, elles sont amenées à désigner les mêmes choses par la nature du matériel filmique (hormis dans le cas du film non narratif et dans le cadre de la narration écrite ou parlée dans laquelle les deux dimensions sont isolables l'une de l'autre). Aussi, Metz prône-t-il une fusion des deux dimensions là où le matériel filmique les confond (p. 186).

Le troisième volet découle de toute l'analyse qui précède et se veut une synthèse théorique qui clôt le travail d'investigation sur l'énonciation. L'énonciateur filmique y est défini comme une entité fondamentalement apersonnelle, toujours hors texte, une entité dont on ne peut rien dire sinon qu'elle a fait l'acte d'énoncer un film ou un texte (p. 189). Conséquence de cette apersonnalisation, Metz rejette les thèses sur l'auteur implicite, auteur modèle et autres qu'il considère comme une prolifération douteuse d'instances au statut suspect (pp.193 et 205) et opte pour une conception fondamentalement apersonnelle dans laquelle l'énonciation est coextensive à tout l'énoncé et n'implique d'autre énonciateur que le film lui-même. L'énonciateur qui dit «je» est donc, par définition, toujours déjà énoncé, toujours inclus dans le film. Malgré cette condamnation inexorable de la conception anthropomorphique et déictique de l'énonciation filmique, Metz évoque cependant la possibilité d'une certaine personnification de l'énonciateur filmique, non pas en terme d'instance énonciative, mais comme principe d'intelligibilité, hypothèse d'interprétation du film (p. 213).

On le voit paraître dans ce trop court survol, L'Énonciation impersonnelle ou le site du film est l'occasion d'une série de mises au point d'une remarquable acuité sur l'énonciation filmique, qui se traduisent par une désinflation du vocabulaire 
théorique. Rompant avec l'opposition énoncé / énonciation, Metz renouvelle de façon brillante la réflexion sur l'énonciation filmique et ouvre la voie à une nouvelle ère théorique qui constitue une écoute subtile du film, une attention à sa saveur énonciative particulière.

Toutefois, s'il m'est permis d'évoquer un doute, on peut se demander si la fusion des concepts de narration et d'énonciation n'a pas un effet réducteur en posant une équivalence pour des figures extrêmement différentes. Je pense notamment aux procédés qui participent du monde diégétique, tels les jeux avec les miroirs, le cadre dans le cadre, certaines voix-Je manifestement destinées à un auditoire diégétique, etc., qui participent du monde narré et dont la valeur énonciative est sujette à des appréciations fort diverses, mais aussi aux relations d'attribution, tel le film dans le film et le récit en voix-Je qui structurent le film en mondes distincts, qui en fixent la structure narrative. N'y aurait-il pas avantage à maintenir la distinction entre énonciation et narration dans la mesure où on peut identifier deux activités distinctes, dans la mesure où elles ont des fonctions différentes même si dans le cadre du film les deux peuvent parfois se confondre au sein de certaines figures? Malgré ce doute, ou plutôt à cause de lui et de tous ceux qu'il peut faire surgir, je ne peux que recommander très vivement L'Énonciation impersonnelle ou le site du film : tous ceux et celles qui s'intéressent aux problèmes de l'énonciation, à la focalisation, à la question du style, etc., y trouveront des réflexions extrêmement stimulantes, des nouvelles pistes de recherche et plus encore, une conception de la théorie cinématographique où la rigueur côtoie l'écoute subtile du film.

Jean Châteauvert Université du Québec à Montréal

\section{NOTES}

1 Christian Metz, «L'Énonciation impersonnelle ou le site du film», Vertigo 1 (1987).

2 La critique de David Bordwell se trouve dans Narration in the Fiction Film (Madison : University of Wisconsin Press, 1985).

3 Michel Chion, La Voix au cinéma (Paris : Éditions de l'Étoile / Cahiers du cinéma, 1982).

4 Edward Branigan, Point of View in the Cinema (New York : Mouton Publishers, 1984).

5 François Jost, L'Eil caméra (Lyon : Presses Universitaires de Lyon, 1987).

6 Marc Vernet, Figures de l'absence (Paris : Éditions de l'Étoile / Cahiers du cinéma, 1988). 